\title{
VOLUMETRIC MAGNETIC RESONANCE IMAGING (MRI) AT TERM PREDICTS MOTOR DEVELOPMENT AT 2 YEARS IN PRETERM INFANTS WITH POST-HEMORRHAGIC VENTRICULAR DILATATION
}

\author{
S. Jary ${ }^{1}$, A. DeCarli ${ }^{2}$, F. Nordio ${ }^{3}$, L. Ramenghi ${ }^{2}$, M. Rutherford ${ }^{4}$, A. Whitelaw ${ }^{1}$ \\ ${ }^{l}$ Clinical Science, University of Bristol, Bristol, UK, ${ }^{2}$ Mother and Infant Sciences, Ospedale Maggiore Cà \\ Granda, Milan, ${ }^{3}$ Clinical Medicine, University of Parma, Parma, Italy, ${ }^{4}$ Robert Steiner MR Unit, Imperial \\ College, London, UK
}

Background: Premature infants with Post-Hemorrhagic Ventricular Dilatation (PHVD) have a high risk of disabilities but prediction of grade of motor and mental outcome is difficult using ultrasound.

Aims: To investigate the correlation of both qualitative and volumetric MRI at term with neurodevelopmental outcome at 2 years.

Methods: 36 premature infants with PHVD, had cerebral MRI at term. MR images were assessedt by two observers unaware of treatment or outcome. Brain regions were assessed and abnormalities scored. In a subgroup of 25 infants, total and regional cerebral volumes were calculated using a manual segmentation technique. Bayley Scales of Infant Development were examined at 2 years past term. As many infants scored below 50 on the Bayley scales, Developmental Quotients (DQ) were calculated from Developmental Age Equivalent scores to provide continuous variables for all.

Results: Qualitative analysis correlated weakly with motor and mental DQ. Total brain volume, excluding ventricles, showed strong positive linear correlation with motor, but not mental DQ (p 0.001).

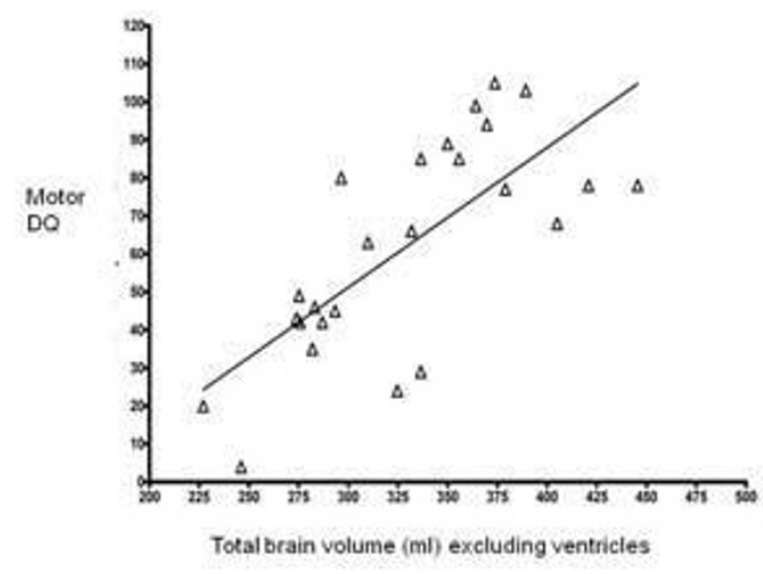

$[M R \operatorname{vol} v D Q]$

Brain volume $<296 \mathrm{ml}$ had $100 \%$ positive predictive value for $\mathrm{DQ}<55$. Thalamic volume and cerebellar volume correlated strongly with motor but not mental DQ. $29.5 \%$ of infants showed normal posterior limb of internal capsule (PLIC) but had mean motor DQ 67.

Discussion: Volumetric brain measurements provided a better prediction of motor development than did qualitative assessment. 